\title{
The Link Between Emotional and Psychological Distress with Myofascial Pain Syndrome
}

\author{
Andrew Lalchhuanawma ${ }^{1, *}$, Divya Sanghi \\ ${ }^{1}$ Department of Physiotherapy, Faculty of Applied Sciences, Manav Rachna International Institute of Research \& Studies, Faridabad, India \\ ${ }^{2}$ Faculty of Applied Sciences, Manav Rachna International Institute of Research \& Studies, Faridabad, India
}

Email address:

Andrewmama182@gmail.com (A. Lalchhuanawma), divya.fas @mriu.edu.in (D. Sanghi)

${ }^{*}$ Corresponding author

To cite this article:

Andrew Lalchhuanawma, Divya Sanghi. The Link Between Emotional and Psychological Distress with Myofascial Pain Syndrome. American Journal of Sports Science. Vol. 7, No. 4, 2019, pp. 177-181. doi: 10.11648/j.ajss.20190704.18

Received: November 4, 2019; Accepted: November 25, 2019; Published: December 2, 2019

\begin{abstract}
Myofascial pain syndrome (MPS) is a common soft tissue disorder estimated to affect more than three-fourths of the general American population at least once during their lifetime. In this day of age, depression is remarkably the leading cause of disability affecting the mind and the emotional state of health. Depression is a serious mental state affecting more than 300 million people worldwide. Depression is said to be associated with chronic myofascial pain due to its dual neuronal connection, both believed to have been influenced by serotonin and nor-epinephrine. As a result, treatment goals should not be directed on the emotional symptoms alone but emphasis should be laid on the physical symptoms as well. Primary healthcare providers should emphasize the intensity and extent of physical symptoms associated with soft tissue disorders. A depressive mood disorder is more often than not associated with physical pain, as is the case with a number of patients presenting at primary care. These patients seek treatment for physical symptoms alone, making depression even harder to diagnose. Depressive mood and physical pain are closely interlinked with each other. Medical intervention with antidepressants that act as both serotonin and norepinephrine reuptake inhibitors in correcting chemical imbalances are indicated for alleviating physical symptoms in depressed individuals.
\end{abstract}

Keywords: Myofascial Pain Syndrome, Depression, Neurotransmitters, Emotional Distress, Psychological Distress

\section{Introduction}

Myofascial pain syndrome is a common soft tissue disorder associated with the presence of taut knot in the muscle fiber known as trigger points. It is estimated to affect $10 \%$ of the general American population and up to $85 \%$ of the population at least once during their lifetime $[1,2]$. The term is often used synonymously with complex regional pain syndrome (CRPS) but differs at length with widespread soft tissue disorder like fibromyalgia syndrome (FMS) and chronic fatigue syndrome (CFS) [3].

Myofascial Pain Syndrome (MPS) doesn't fall under the sub-category of emotional or psychological disorder as it is a widely known regional musculoskeletal disorder. Yet, there exists a strong correlation between myofascial pain with the emotional aspects of depression, anxiety, sleep deprivation and stress along with the physical state of an individual $[4,5]$.
Moldofsky and Munoz et al. concluded in their study that sleep quality corresponds with an intensity of pain $[6,7]$.

It is further considered that individuals with sleep disturbances associated with soft tissue injury affect daily work consequently delaying their return to work. First, let us consider a list of certain emotional and physical factors accountable for aggravating myofascial pain syndrome [8]. These are;

Emotional

1. Depression Anxiety disorder- panic, tension, stress, Post Traumatic Stress Disorder (PTSD).

2. Mood disorder- bipolar (manic-depression), mood swings.

3. Cognitive dysfunction- memory, learning and reasoning skills, intellectual task, Attention Deficit Hyperactivity Disorder (ADHD).

4. Sleeping disorder- insomnia, sleep apnea.

Physical 
1. Aches- back, stomach, joint, muscle.

2. Muscle tension and stress.

3. Fatigue (muscle weakness).

4. Muscle pain (widespread/regional musculoskeletal pain).

5. Degenerative diseases- Parkinson's, Moto- neurones, Multiple sclerosis.

In today's world, depression undoubtedly is the leading cause of disability which affects the emotional state of health and wellness [9]. The growing trend of depression could only be anticipated to rise further in no time. Even as we speak, it has already taken its toll in the global general population. According to the World Health Organization (WHO) 2018, depression is a serious mental disorder affecting more than 300 million people worldwide. It is found that females have higher rates of depression as compared to their male counterpart. A bout of depression could result in taking extreme steps like contemplating suicide and/or suicidal tendencies. Depression accounts for nearly 800,000 deaths worldwide due to suicide alone [9].

The rate of suicidal tendency is found to be higher in patients with chronic pain [8]. Ohayon and Schatzberg analyzed the general population in terms of their depressive morbidity [10]. It was reported that $33 \%$ or 687 individuals who had suicidal tendencies were also found to have a chronic pain condition. Fishbain in his literature review concluded that individuals who contemplated suicide, had suicidal tendencies or suicidal thoughts are commonly associated with chronic pain. The author further added that chronic pain may be one of the risk factors for developing suicidal tendencies [11].

Psychological distress or mental distress usually resulting from an unwanted experience may trigger symptoms such as depression, tensor stress, and panic attack due to Post Traumatic Stress Disorder (PTSD). It is described as an unpleasant feelings or emotions impacting the levels of normal functioning. Individuals with emotional distress may show signs of a feeling of anxiety, panic, tension stress, insomnia, depression, and other self-destructive thoughts [8].

\section{Depression and Chronic Myofascial Pain}

The common presenting physical symptoms of depression include achiness of the joints, achiness of muscles of the back and the extremities, muscle tension and stress, muscle fatigue, gastrointestinal disturbance, sleep apnea and altered psychomotor activities [8]. These physical signs in depression may progressively develop into chronic pain, owing to the distress of muscle tension. As a result, depressed individuals are more likely to develop pain because they are more aware of their chronic physical pain.

The tense muscle fibers translate into a taut band resulting in the formation of myofascial trigger points. Depression and other emotional distress like stress, tension and anxiety are often expressed in the form of muscle tension. These tense muscle fibers are held into sustained contraction causing muscle overload, thus provoking the perpetuation of myofascial trigger points (MTrPs). In general, individuals having these physical signs and problems are usually unaware of the presence of their tense muscles [1].

At some point in time, it is safe to assume that individuals encounter at least a dose of stressful life events. The emotional stress could be in the form of losing loved ones, trauma, bankruptcy, divorce, etc. The stressful past events keep resonating throughout life, orchestrating physiological changes. This mechanism cascaded the stress hormone in the region of the brain involved in stress mechanism [8].

Simon et al. conducted a study on the relationship between somatic symptoms and depression [12]. They concluded that of the 1146 patients diagnosed with depression, a staggering $69 \%$ of patients cited physical symptoms as the reason for their visit. Ohayon and Schatzberg suggest a systemic approach for health care providers to assess for signs of depression in patients presenting with chronic pain patients [10].

Depression, being the byproduct of chronic pain, is also closely associated with myofascial pain. M. Okumus et al. reported higher levels of depression in patients with myofascial pain syndrome when compared to controls consisting of healthy individuals. They also found a positive correlation between levels of pain intensity with levels of depression using the Beck Depression Inventory (BDI) questionnaire [13].

As the intensity of pain is higher, the level of depression in an individual is more likely to be greater. The degree of developing emotional symptoms is greater with an increase in the number of physical symptoms. A study conducted by Kroenke at al. established the severity of depression to be directly correlated with the number of physical symptoms. It was reported that $60 \%$ of individuals found to have depression presented with nine or more physical signs or symptoms, whereas $2 \%$ of those individuals diagnosed with depressive disorder were found to have only one or no physical symptoms [14].

\section{Dual Neuronal Connection}

The relationship between depression and physical pain can be attributed to a complex mechanism involved in the neurochemical pathway in that both are influenced by neurotransmitters like serotonin and norepinephrine. Both the physical aspect of pain and the emotional aspect of depressive mood share the same neural pathway [8, 15]. It is to be understood that the human brain alone is estimated to be made up of 100 billion neurons, creating 100 trillion neural connections.

Neurons are specialized cells or units of the nervous system capable of transmitting nerve impulses. They receive and sends a signal from the body to the brain and back to the body. Neurons communicate with each other through a combination of electrical and chemical signals using special projections called axons and dendrites. The electrical signal travels down 
the neuron along the length of the axon to its terminal end (axon terminal) where it is converted into a chemical signal. The signal releases chemical messengers known as neurotransmitters into the space between the neuron and the adjoining tip of dendrite from another neuron [12].

The space or junction between the two neurons is called synapse. From the synapse, neurotransmitters are received by the neighboring neuron and convert them back into electrical signals. These neurotransmitters or chemical messengers are usually made up of amino acids. It is of paramount importance to note that amino acids are the building blocks of protein. Proteins are the essential nutrients required to maintain the desired levels of neurotransmitters and are also required for the normal functioning of the brain and the body. Any interruption in communication could potentially result in serious health consequences on the emotional, psychological and physical well-being of individuals. Therefore, imbalances in the levels of neurotransmitters could probably affect mood behavior and other health issues.

Neurotransmitters send chemical messages or signals from one neuron (nerve fiber) to another. These chemicals bind with their respective matched receptors located at target sites of the brain and the body. It acts by regulating vast processes involved in the perception of pain and the Limbic System in particular, which controls the emotional aspects of depression, mood, memories, and pleasure known to directly relate with chemical imbalances. The link between depression and widespread musculoskeletal pain can be attributed to dysregulation of the neurotransmitters $[8,16]$.

\section{Neurotransmitters}

The main neurotransmitters involved in the normal functioning of the brain and the body is as follows [16];

1. Inhibitory (calming) neurotransmitters: Acetylcholine (Ach), Serotonin (5-HT) and Gamma Amino Butyric Acid (GABA).

2. Excitatory (stimulating) neurotransmitters: Norepinephrine, Dopamine and Glutamate.

Acetylcholine is both an inhibitory and excitatory neurotransmitters and acts both in the Peripheral Nervous System (PNS) and Central Nervous System (CNS). It is excitatory at the neuromuscular junction (NMJ), at the skeletal muscle and causes a muscle to contract.

Upon noxious stimulation or pain, the sensory nociceptive fibers enter the central nervous system (CNS) through the dorsal horn of the spinal cord where it synapses with the spinal neurons. Neurons project along the spinothalamic and spinoreticulo-thalamic tracts to the thalamus, parabrachial nucleus, and amygdala where they are further processed to higher cortical centers. Descending transmission of pain is modulated by several mechanisms including the neurotransmitters viz. serotonin (5-HT, Tryptophan hydroxylase), nor-epinephrine (Tyrosine hydroxylase) and dopamine which affects the behavioral aspects of mood and emotions [17].

Even if the acute emotional symptoms of depression have subsided, symptoms of physical pain should be considered in the treatment plan to achieve full recovery. In such a case, it is, therefore, advisable to administer antidepressant medication precariously that influences both pain and depression. Serotonin-norepinephrine reuptake inhibitors are examples that act by combating emotional symptoms in depressed individuals who present with physical symptoms. Any psychotropic medication should be administered with the help of a certified, skilled practitioner on the recommended dosage to avoid the adverse effects. There have been numerous reports suggesting the efficacy of this type of dual action antidepressants comparing with the selective serotonin reuptake inhibitors [8].

\section{Regions Regulating Mood}

A certain area of the brain helps in regulating depressive mood [16]. New and sophisticated imaging technologies for mapping the brain have been developed over the years which were not accessible in the recent past. The fMRI (functionalMagnetic Resonance Imaging) can detect changes in parts of the brain while performing tasks. On the other hand, Positron Emission Tomography (PET) and Single Photon Emission Computed Tomography (SPECT) can map the brain by recording the distribution and density of neurotransmitters. It helps in a better understanding of the areas affected by depression i.e. the Thalamus and the Limbic System comprising the Amygdala and Hippocampus.

The areas of the brain mainly involved in depression are as follows [16];

i. Amygdala- it is part of the limbic system which controls the emotional aspect of anger, pleasure, sexual arousal, sorrow, fear. This part of the brain is triggered when a person is haunted by an uneventful experience, recollecting an extreme frightening state of emotions.

ii. Thalamus- it first receives sensory information and relays this information to a higher cortical center into the cerebral cortex. It controls the higher cognitive functions such as thinking, learning, reasoning, speech, movement. Research suggests impairing functions of the thalamus to be related to Bipolar disorder.

iii. Hippocampus- it is part of the limbic system which controls the long term memory. Research suggests the hippocampus to be smaller in depressed person and stress hormones like a corticotropin-releasing hormone (CRH) and adreno-cortico-tropic hormone (ACTH) impair the growth of neurons in depression. Evidence suggests a $10-13 \%$ reduction in hippocampal volumes in individuals with a history of depression compared with healthy subjects [18].

\section{Chronic Medical Illness}

Medical conditions account for over $10-15 \%$ of all patients diagnosed with depression [16]. The commonly observed medical case is a thyroid hormone imbalance. Hyperthyroidism (excess thyroid) is often connected with manic disorders, whereas hypothyroidism (little thyroid) often 
leads to lethargy and depression. Certain chronic diseases are linked to depression and mood disorders [16].

The most common non-communicable diseases like cardiovascular disease, diabetes, stroke, and cancer are significantly related to depression [20]. Depressed individuals, especially with a history of heart attacks were given approximately 6 months to live. Among other medical conditions, systemic disease, neuro-degenerative medical conditions and nutritional deficiencies like vitamin B12 have also been associated with depression [1].

\section{Conclusion}

Checklists are organized in individuals for a range of emotional and psychological distress in individuals with soft tissue disorders (chronic musculoskeletal pain or myofascial pain) [1]. These are; identifying the primary source of their emotional distress (depression, stress, tension, anxiety), adopting the necessary lifestyle changes to abate depressive mood disorders, and cross-examining the perpetuating factors for lasting relief.

Primary healthcare providers should emphasize the intensity and extent of physical symptoms associated with soft tissue disorders. After all, a depressive mood disorder is associated with physical pain. A large number of patients who present at primary care seek treatment for physical symptoms alone, making depression even harder to diagnose. Depressive mood and physical pain are closely interlinked with each other, as they share the same neuronal pathway and are modulated by neurotransmitters like serotonin and nor- epinephrine.

As a result, treatment goals should not only be directed on the emotional symptoms but to accentuate on the physical symptoms as well. For alleviating symptoms of physical pain, anti-depressants that act as both serotonin and norepinephrine reuptake inhibitors in correcting chemical imbalances is indicated in depressed individuals [8, 19].

\section{Conflict of Interest}

The authors declare that they have no competing interests.

\section{Funding or Grants}

The authors gained no financial benefit and no funding was received for the study.

\section{References}

[1] Simons, D. G., Travell, J. D. and Simons, L. S, Travell and Simons' Myofascial pain and dysfunction: The trigger point manual vol. 1. Upper half of body (2nd edition), Baltimore MD: Williams and Wilkins, 1999.

[2] Andrew Lalchhuanawma, "Myofascial Pain Syndrome: Physical Activity, Nutrition and Health." American Journal of Sports Science and Medicine, vol. 7, no. 1 2019: 20-22.

[3] Andrea L. Nicol, Matthew Crooks, Eric S. Hsu, F. Michael
Ferrante. Chapter 25 - Myofascial Pain Syndrome. Essentials of Pain Medicine (Fourth Edition). Elsevier Inc., 2018, Pages 207-212.

[4] Ge HY, Fernandez-de-Las-Penas C, Arendt-Nielsen L. "Sympathetic facilitation of hyperalgesia evoked from myofascial tender and trigger points in patients with unilateral shoulder pain." Clinical Neurophysiology, 2006; 117: 1545-50.

[5] Yap EC. Annals Academy of Medicine. "Myofascial Pain - An Overview." Annals of the Academy of Medicine, Singapore, 2007; 36: 43-8.

[6] Harvey Moldofsky. "Sleep and pain.” Sleep Medicine Reviews, Vol. 5, No. 5, pp 387-398, 2001.

[7] Munoz-Munoz S, Munoz-Garcia MT, Alburquerque- Sendin F, Arroyo-Morales M, Fernandez-de-las-Penas C. "Myofascial trigger points, pain, disability and sleep quality in individuals with mechanical neck pain." Journal of Manipulative and Physiological Therapeutics. 2012; 35 (8): 608-13.

[8] Madhukar H. Trivedi. "The link between Depression and Physical Symptoms." The Primary Care Companion to the Journal of Clinical Psychiatry, 2004; 6 (suppl 1): 12-16.

[9] World Health Organisation. 2018, Depression, https://www.who.int/news-room/fact sheets/detail/depression.

[10] Ohayon MM, Schatzberg AF. "Using pain to predict depressive morbidity in the general population." Archives of General Psychiatry, 2003; 60: 39-47.

[11] Fishbain DA. "The association of chronic pain and suicide." Seminars in Clinical Neuropsychiatry, 1999; 4: 221-227.

[12] Simon GE, Von Korff M, Piccinelli M, et al. "An international study of the relation between somatic symptoms and depression." The New England Journal of Medicine, 1999; 341: 658-659.

[13] Okumus, M., Ceceli, E., Tuncay, F., Kocaoglu, S., Palulu, N. and Yorgancioglu, Z. R, "The relationship between serum trace elements, vitamin B12, folic acid and clinical parameters in patients with myofascial pain syndrome.” Journal of Back and Musculoskeletal Rehabilitation, 23 (4), 187-191, Nov. 2010.

[14] Kroenke K, Spitzer RL and Williams JB. "Physical symptoms in primary care: predictors of psychiatric disorders and functional impairment." Archives of Family Medicine, 1994; 3: 774-779.

[15] Basbaum AI and Fields HL. "Endogenous pain control mechanisms: review hypothesis." Annals of Neurology, 1978; 4: $451-462$

[16] What causes depression? - Harvard Health. https://www.health.harvard.edu/mind-and-mood/what-causesdepression.

[17] Barbara Cagnie, Vincent Dewitte, Tom Barbe, Frank Timmermans, Nicolas Delrue and Mira Meeus. "Physiologic Effects of Dry Needling." Current Pain and Headache Reports, 2013; 17: 348 .

[18] Yvette I. Sheline, Milan Sanghavi, Mark A. Mintun and Mokhtar H. Gado. "Depression Duration But Not Age Predicts Hippocampal Volume Loss in Medically Healthy Women with Recurrent Major Depression.” Journal of Neuroscience, 15 June 1999; 19 (12) 5034-5043.

[19] Stahl SM. "Does depression hurt? [BRAINSTORMS]." The Journal of Clinical Psychiatry, 2002; 63: 273-274. 
[20] Boutayeb, A., Boutayeb, S., \& Boutayeb, W. "Multi-morbidity of non-communicable diseases and equity in WHO Eastern Mediterranean countries." International journal for equity in health, 2013; 12, 60 . 